\title{
Glutamate Transporter GLT-1 Is Transiently Localized on Growing Axons of the Mouse Spinal Cord before Establishing Astrocytic Expression
}

\author{
Keiko Yamada, ${ }^{1}$ Masahiko Watanabe, ${ }^{1}$ Takashi Shibata, ${ }^{2}$ Masabumi Nagashima, ${ }^{1}$ \\ Kohichi Tanaka, ${ }^{3}$ and Yoshiro Inoue ${ }^{1}$ \\ Departments of ${ }^{1}$ Anatomy and 2 Urology, Hokkaido University School of Medicine, Sapporo 060-8638, Japan, and \\ ${ }^{3}$ Department of Degenerative Neurological Diseases, National Institute of Neuroscience, National Center of Neurology and \\ Psychiatry, Kodaira 187-8502, Japan
}

The glutamate transporter GLT-1 is expressed in astrocytes of the mature brain and spinal cord. In the present study, we examined its expression in the developing mouse spinal cord. By in situ hybridization, ${ }^{35} \mathrm{~S}$-labeled antisense oligonucleotide probes for GLT-1 mRNA consistently labeled the mantle zone/ gray matter from embryonic day 11 through the adult stage. However, immunohistochemistry with a specific antibody visualized distinct regional and cellular localizations during the time between the fetal and postnatal stages. At fetal stages, GLT-1 immunoreactivity predominated in the marginal zone/white matter, observed as tiny puncta in cross-sections and as thin fibers in longitudinal sections. The GLT-1-immunopositive structures were also labeled for neuron-specific enolase, a glycolytic enzyme specific to postmitotic neurons and endocrine cells. By electron microscopy, GLT-1 immunoreactivity was detected in axons forming frequent enlargements and was focally localized on a small portion of the axolemma, particularly that facing adjacent axons. At early postnatal stages, GLT-1 disappeared from axons in white matter tracts and, instead, appeared in astrocytic processes surrounding various neuronal elements in the gray matter. Therefore, before switching to astrocytic expression, GLT-1 is transiently expressed in neurons and localized in differentiating axons. Together with our previous finding on the localization of glutamate transporter GLAST in radial glial fibers, GLT-1 and GLAST are thus localized during development on distinct directional cellular elements along which young neurons elongate their axons or move their cell bodies, respectively.

Key words: glutamate transporter; GLT-1; mouse; spinal cord; growth cone; astrocyte; immunoblot; immunohistochemistry; in situ hybridization; electron microscopy; development
Glutamate is a major neurotransmitter mediating the fast excitatory transmission at central synapses and plays critical roles in synaptic plasticity and development (Mayer and Westbrook, 1987; Monaghan et al., 1989). The extracellular glutamate concentration has to be kept low enough to terminate glutamate receptor activation and to protect neurons from glutamate excitotoxicity (Herz, 1979; Choi, 1992). The low extracellular concentration is attributable to the action of high-affinity sodium-dependent glutamate transporters. Molecular cloning studies have identified several subtypes of the glutamate transporter with distinct structures, functions, and expressions (Kanai et al., 1997). Of these, GLT-1 and GLAST are astrocytic glutamate transporters in the adult CNS; the former is highly expressed in the telencephalon, and the latter predominates in the cerebellum (Kanai and Hediger, 1992; Storck et al., 1992; Tanaka, 1993; Rothstein et al., 1994; Torp et al., 1994; Lehre et al., 1995; Shibata et al., 1996). These brain regions are known to receive massive glutamatergic inputs. Both transporters are abundantly localized on the astrocytic cell

Received March 13, 1998; revised May 11, 1998; accepted May 13, 1998.

This investigation was supported by research grants to M.W. from the Ministry of Education, Science, Sports, and Culture, the Ministry of Health and Welfare, the Science and Technology Agency (Strategic Promotion System for Brain Science), Naito Foundation, Suhara Foundation, and Kanahara Foundation and to K.Y. from the Research Fellowships of the Japan Society for the Promotion of Science for Young Scientists.

Correspondence should be addressed to Masahiko Watanabe, Department of Anatomy, Hokkaido University School of Medicine, Sapporo 060, Japan.

Copyright (C) 1998 Society for Neuroscience $\quad 0270-6474 / 98 / 185706-08 \$ 05.00 / 0$ membrane, particularly that surrounding synapses (Chaudhry et al., 1995). Inactivation of the GLT-1 gene results in a prolonged presence of glutamate in the synaptic cleft and causes neuronal degeneration in the hippocampus, whereas that of the GLAST gene increases the vulnerability to acute cerebellar injury (Watase et al., 1998; Tanaka et al., 1997). All of the evidence indicates important roles of astrocytic glutamate transporters in the clearance of synaptically released glutamate and the protection of neurons from glutamate excitotoxicity.

From early stages before synaptogenesis, GLAST and GLT-1 mRNAs are also expressed, showing different spatial distributions from each other (Shibata et al., 1996; Sutherland et al., 1996). In mice, cells expressing GLAST mRNA are restricted to the ventricular zone at embryonic day 13 (E13) and then appear and spread over the mantle zone/gray matter. Recently, we have demonstrated in the mouse spinal cord that GLAST is expressed in the radial glia-astrocyte lineage (Shibata et al., 1997); radial glia comprises a distinct cell class of neuroglia, which guides neuronal migration and transforms later into astrocytes and oligodendrocytes (Rakic, 1971; Choi, 1981; Raff et al., 1983). On the other hand, GLT-1 mRNA is primarily expressed in the mantle zone/gray matter from early brain development. To understand the cellular and molecular system for glutamate transporters in the developing CNS, we examined the mouse spinal cord by in situ hybridization using ${ }^{35}$ S-labeled antisense oligonucleotide probes and by immunohistochemistry with affinity-purified specific antibodies. We report here that GLT-1 exhibits transient 
neuronal expression and axonal localization before its astrocytic expression is established.

\section{MATERIALS AND METHODS}

Antibody. To raise polyclonal antibodies in rabbits and guinea pigs, several $\mathrm{N}$ - and $\mathrm{C}$-terminal sequences of the mouse GLT-1 were chosen for the antigen. The amino acid sequences of three synthetic peptides, which are predicted to be intracellular, were MASTEGANNMP KQVEVRMHDSHLSSDEP, LDTIDSQHRMQEDIEMTKTQSIY DDK, and KSADCSVEEEPW KREK, which correspond to amino acid residues 1-28 (termed GLT/3), 500-525 (GLT/1), and 557-572 (GLT/5) of the mouse GLT-1, respectively (Mukainaka et al., 1995). A cysteine residue was introduced at the $\mathrm{C}$ or $\mathrm{N}$ terminus of the first two peptides to facilitate the conjugation to keyhole limpet hemocyanin with the 3-maleidobenzoic acid $N$-hydroxysuccinimide ester method, whereas the latter was coupled to thyroglobulin with glutaraldehyde. In addition, amino acid residues 1-73 (GLT/9) were expressed as a glutathione $S$-transferase fusion protein, using the pGEX-4T-2 plasmid vector (Pharmacia, Uppsala, Sweden). The fusion protein was purified with glutathione-Sepharose 4B, cleaved by thrombin, and purified by reversephase HPLC, as described previously (Watanabe et al., 1998). Antigen peptides were emulsified with Freund's complete adjuvant (Difco, Detroit, MI) and injected subcutaneously into New Zealand white rabbits and Hartley guinea pigs at intervals of 2-4 weeks. From antiserum sampled 2 weeks after the sixth injection, the $\operatorname{IgG}$ fraction was purified using protein G-Sepharose (Pharmacia). GLT-1-specific IgG was then affinity-purified using synthetic peptides or fusion protein coupled to cyanogen bromide-activated Sepharose 4B (Pharmacia).

In the present study, we also used a guinea pig anti-GLAST polyclonal antibody, whose specificity has been reported previously (Shibata et al., 1997). Rabbit polyclonal antibody against human neuron-specific enolase (NSE) was purchased from Dako (Carpinteria, CA), and the specificity was reported previously (Watanabe et al., 1990).

Immunoblot. Membrane extracts from the adult C57BL mouse brain were prepared as described previously (Yamada et al., 1996). Seven micrograms of protein were analyzed by $11 \%$ SDS-PAGE under reducing conditions. Proteins in the gel were electroblotted onto a nitrocellulose membrane, incubated with $1 \mu \mathrm{g} / \mathrm{ml}$ affinity-purified antibodies in PBS containing $0.1 \%$ Tween 20 , and visualized with the ECL detection system (Amersham, Arlington Heights, IL).

In situ hybridization. Under deep pentobarbital anesthesia, the lumbar cord was freshly sampled from C57BL mice at E11, E13, E15, E18, postnatal day 1 (P1), P7, P14, P21, and 4 months (adult) and frozen in powdered dry ice. The next day of overnight mating was counted as E0. Fresh frozen sections were prepared by cryostat $(20 \mu \mathrm{m}$ thick) and processed for in situ hybridization. The sequence of two nonoverlapping antisense oligonucleotide probes and the procedures for in situ hybridization were the same as reported previously (Shibata et al., 1996). Briefly, hybridization was performed overnight with $10,000 \mathrm{dpm} / \mu 1^{35} \mathrm{~S}$ labeled probes at $42^{\circ} \mathrm{C}$ in the presence of $50 \%$ formamide, followed by washing in $0.1 \times \mathrm{SSC}$ containing $0.1 \%$ sarcosyl at $55^{\circ} \mathrm{C}$. Sections were exposed to nuclear track emulsion (NTB-2; Kodak, Rochester, NY) for 2 months.

Immunohistochemistry. For immunohistochemistry, fetuses at E11, $\mathrm{E} 13, \mathrm{E} 15$, and E18 were fixed at $4^{\circ} \mathrm{C}$ by overnight immersion in Bouin's fixative for paraffin sections or in $4 \%$ paraformaldehyde in $0.1 \mathrm{~m}$ sodium phosphate buffer (PB), pH 7.2, for cryostat sections, whereas mice at P1, P7, P14, P21, and adult (4 months old) were all perfused transcardially with the latter fixative under deep pentobarbital anesthesia. As a specificity control, GLT-1 $(-/-)$ mice at E13 and the adult stage (Tanaka et al., 1997) were similarly fixed as above. Paraffin $(5 \mu \mathrm{m})$ and cryostat sections $(20 \mu \mathrm{m})$ were prepared and incubated overnight with GLT-1 antibodies $(0.1-0.3 \mu \mathrm{g} / \mathrm{ml})$ at room temperature. Sections were then incubated with biotinylated goat anti-rabbit IgG for $1 \mathrm{hr}$ and streptavidin for 30 min, using a Histofine SAB-PO(R) kit (Nichirei Corp., Tokyo, Japan). Immunoreaction was visualized with 3,3'-diaminobenzidine. For immunoelectron microscopy, immunostained sections were further treated with osmium tetroxide and uranyl acetate, dehydrated, and embedded in Epon 812.

For double labeling for GLT-1 and GLAST, paraffin sections were first processed for rabbit anti-GLT-1 antibody $(0.3 \mu \mathrm{g} / \mathrm{ml})$ and Histofine SAB-PO(R) kit, followed by visualization with the Tyramide signal amplification kit [TSA-DIRECT (Green); NEN Life Science, Boston, MA]. The second immunoreaction was done with guinea pig antiGLAST antibody $(2.5 \mu \mathrm{g} / \mathrm{ml})$ and phosphatase-linked anti-guinea pig
IgG (Kirkegaard \& Perry, Gaithersburg, MD) and visualized with the HNPP fluorescent detection set (Boehringer Mannheim, Mannheim, Germany). For double labeling for GLT-1 and NSE, cryostat sections were first incubated with guinea pig anti-GLT-1 antibody $(1.0 \mu \mathrm{g} / \mathrm{ml})$, biotinylated goat anti-guinea pig IgG (1:200) (Vector Laboratories, Burlingame, CA), and streptavidin-peroxidase, followed by visualization with TSA-DIRECT (Red). The second immunoreaction was done with rabbit anti-NSE antibody (1:3000) and FITC-labeled donkey anti-rabbit IgG (1:100) (Jackson ImmunoResearch, West Grove, PA). Sections were photographed by a confocal laser scanning microscope (MRC 1024; Bio-Rad, Hercules, CA).

Electron microscopy. Mice at E13 were immersed overnight in $4 \%$ paraformaldehyde in $0.1 \mathrm{M} \mathrm{PB}, \mathrm{pH} 7.2$, and post-fixed for $2 \mathrm{hr}$ with $1 \%$ osmium tetroxide in $0.1 \mathrm{M} \mathrm{PB}$. After block staining in $1 \%$ aqueous uranyl acetate solution and dehydration, tissue specimens were embedded in Epon 812. Silver-gold ultrathin sections were prepared on an Ultracut ultramicrotome (Leica, Nussloch, Germany) and stained with $1 \%$ uranyl acetate for $5 \mathrm{~min}$ and a mixed lead solution for $2 \mathrm{~min}$. Electron micrographs were taken on an H7100 electron microscope (Hitachi, Tokyo, Japan).

\section{RESULTS}

\section{In situ hybridization}

By in situ hybridization with ${ }^{35} \mathrm{~S}$-labeled antisense oligonucleotide probes, developmental changes of GLT-1 mRNA expression were examined in cross-sections of the mouse lumbar cord from E11 to the adult stage (Fig. 1). At E11, low levels of GLT-1 mRNA were detected in a thin outer layer (the mantle zone), whereas no significant signals were found in the large central region (the ventricular zone) (Fig. $1 A$ ). In good accordance with the marked expansion of the mantle zone with concomitant reduction of the ventricular zone and central canal, GLT-1 mRNA became detectable in most regions of the spinal cord by E15 (Fig. 1B,C). In postnatal development, levels of GLT-1 mRNA exhibited a prominent increase in the gray matter, peaking at P14 (Fig. $1 D-F$ ). Weaker signals were also detected in the white matter at P14 and thereafter. Almost identical results were obtained with another nonoverlapping antisense probe (data not shown).

\section{Antibody preparation}

Using $\mathrm{N}$ - and C-terminal peptides as antigens, four polyclonal antibodies against the mouse GLT-1 were obtained in rabbits and guinea pigs. By immunoblot analysis, all of these antibodies recognized a $60-70 \mathrm{kDa}$ band in the brain of the adult wild-type mouse (Fig. 2A). With C-terminal antibodies, the band was diminished in intensity in the GLT-1 $(+/-)$ mouse brain and disappeared in the GLT-1(-/-) mouse brain. Immunohistochemistry with microslicer sections showed that each antibody widely stained the adult brain and showed the highest levels in the telencephalon, including the cerebral cortex, hippocampus, caudate-putamen, and olfactory tubercle (Fig. 2B). The distribution of GLT-1 protein was similar to that of GLT-1 mRNA (Fig. $2 C$ ). In the adult spinal cord, each antibody stained neuropil regions of the gray matter, higher in the dorsal horn than in the ventral horn (Fig. 2D). The results from immunoblotting and immunohistochemistry are consistent with previous reports (Rothstein et al., 1994; Lehre et al., 1995). Of the four antibodies, the strongest immunohistochemical signal was given by C-terminal antibodies: rabbit antibody against amino acid residues 557-572 and guinea pig antibody against amino acid residues 500-525. All immunohistochemical data presented here were obtained with the two antibodies.

Preabsorption of antibodies (for example, addition of $7.5 \mu \mathrm{g} / \mathrm{ml}$ antigen peptide at adult and $0.075 \mu \mathrm{g} / \mathrm{ml}$ at E13 to rabbit antibody against amino acid residues 557-572) completely abolished the 

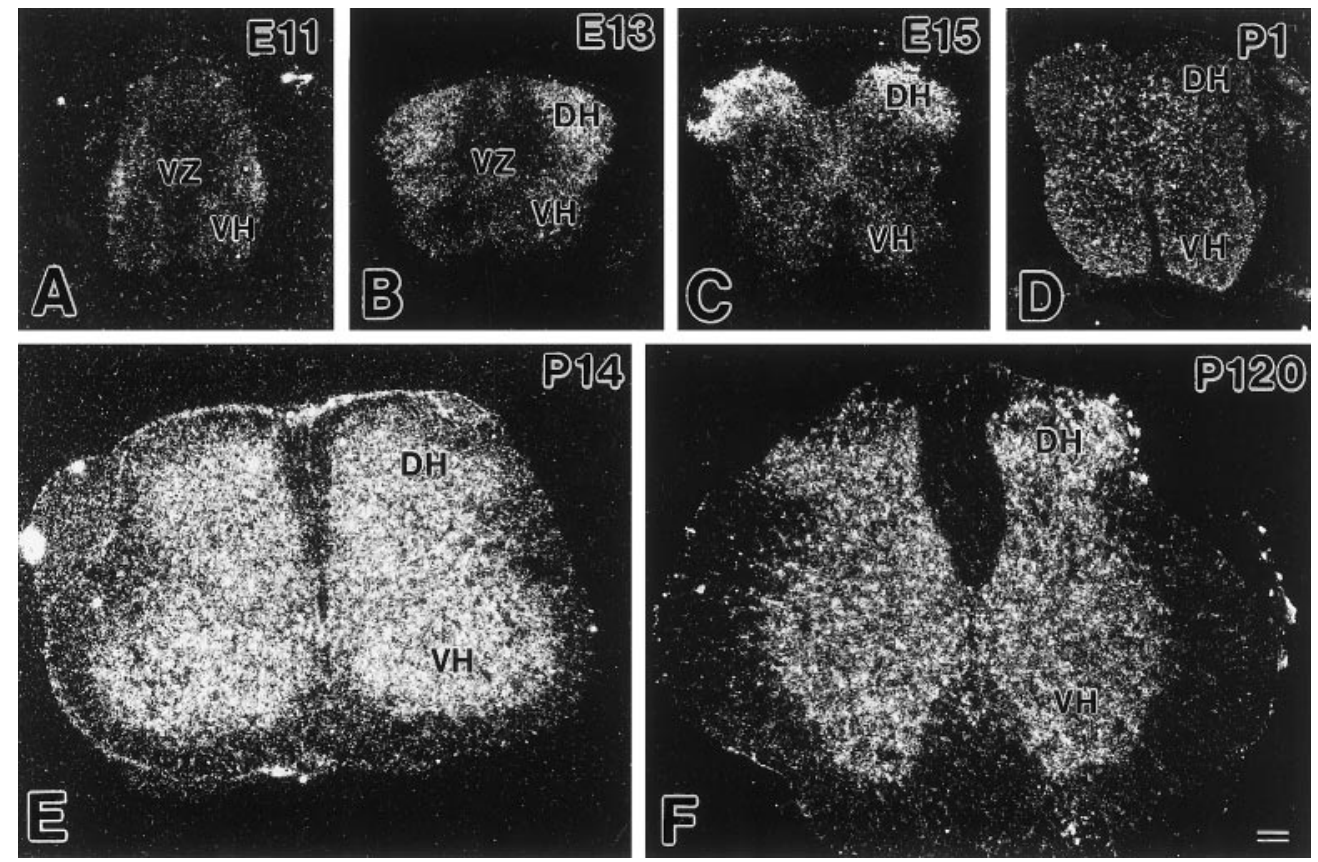

$V H$, ventral horn; $V Z$, ventricular zone. Scale bar, $100 \mu \mathrm{m}$.

Figure 2. Characterization of GLT-1 antibody. $A$, Immunoblot analysis using brain extracts from the adult wild-type $(a)$, GLT- $1(+/-)(b)$, and GLT-1 $(-/-)$ (c) mice. The size of protein markers is $205,116,80$, and $49.5 \mathrm{kDa}$ from the above. $B$, Immunohistochemistry for GLT- 1 in the adult mouse brain. $C$, In situ hybridization for GLT-1 mRNA in the adult mouse brain. $D$, Immunohistochemistry for GLT-1 in the adult mouse spinal cord. $C b$, Cerebellum; $C P$, caudate-putamen; $C x$, cerebral cortex; $\mathrm{DH}$, dorsal horn; $\mathrm{Di}$, diencephalon; $\mathrm{Hi}$, hippocampus; $M b$, midbrain; $M O$, medulla oblongata; $P o$, pons; $T u$, olfactory tubercle; $V H$, ventral horn. Scale bars: $B, C, 2 \mathrm{~mm} ; D, 100 \mu \mathrm{m}$.
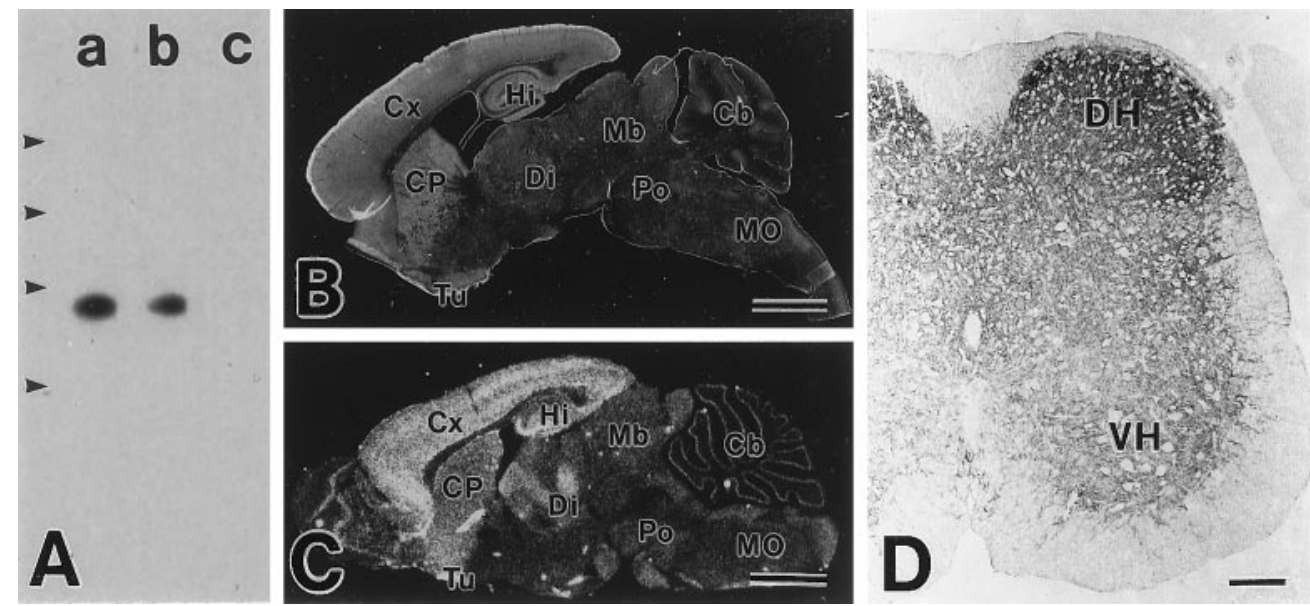

characteristic staining in the spinal cord (data not shown). Under the same conditions, $\mathrm{C}$-terminal antibodies yielded no significant immunohistochemical signals in the adult spinal cord of the GLT-1(-/-) mouse (data not shown). Therefore, immunohistochemical signals by the present antibodies were judged to be specific to GLT-1.

\section{Immunohistochemistry}

Developmental changes at the protein level were followed by light-microscopic immunoperoxidase (Fig. 3, see Fig. 6A-E,G), immunofluorescence (Fig. 4), and immunoelectron microscopy (Figs. 5B, 6F). At E11 when the mouse spinal cord consists of a large ventricular zone, a small oval bulging of the ventral horn, and a thin peripheral layer of the marginal zone (Shibata et al., 1997), very weak immunoreactivity was found in the marginal zone/white matter layer (data not shown).

At E13, GLT-1 immunoreactivity in the marginal zone was increased and distributed in the ventral and lateral funiculi (Fig. $3 A, B)$. The mantle zone, on the other hand, was associated with little immunoreactivity, except for labelings scattered in the dorsal horn. No immunoreactivity was detected in the spinal cord of the GLT-1(-/-) mouse (Fig. 3C). At higher magnification, GLT-1 immunoreactivity in the marginal zone was observed as numerous tiny puncta in cross-sections (Fig. 3D) and as fibrous structures running rostrocaudally in longitudinal sections (Fig. $3 E, F)$. By confocal laser scanning microcopy with a high-sensitive fluorescence signal amplification system, the distribution of GLT-1 immunoreactivity was visualized more clearly (Fig. 4A, $B$, green). Within the marginal zone, labeled structures were more numerous and intense in deeper regions than in superficial regions just beneath the pial surface (Fig. 4B). In the mantle zone, there were some transverse fibers that ran toward the marginal zone and showed low GLT-1 immunoreactivity (Fig. 4B, arrows). When compared with glutamate transporter GLAST expressed in radial glial cells (Fig. 4A, B, red), both showed no overlaps; GLT1-immunopositive puncta were all immunonegative for GLAST, and GLAST-immunopositive radial fibers running in the mantle and marginal zones were all immunonegative for GLT-1. By double immunofluorescence for NSE, a soluble glycolytic enzyme specific to postmitotic neurons and endocrine cells (Calker et al., 1978; Watanabe et al., 1993), immunoreactivities for GLT-1 (Fig. 

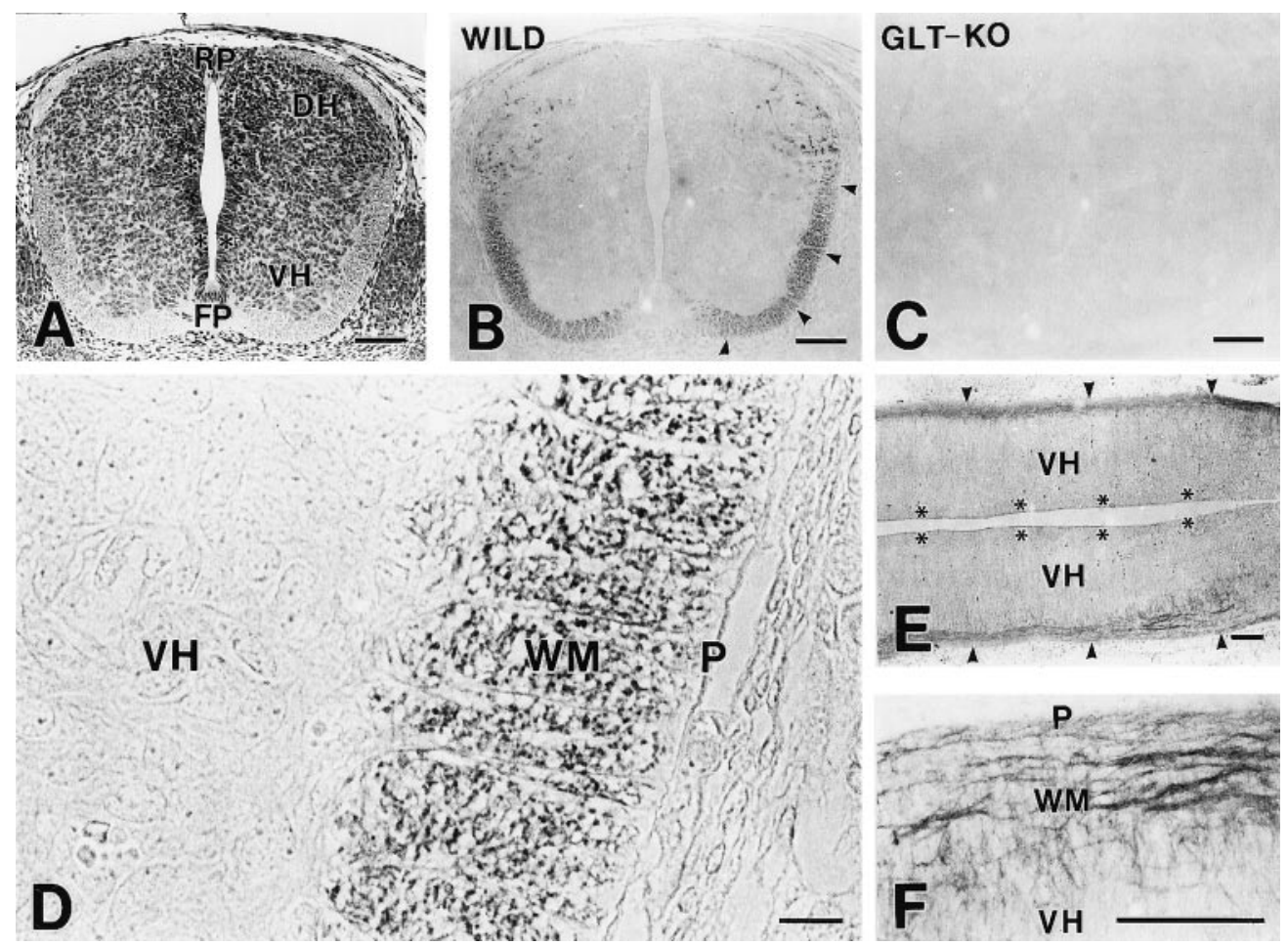

Figure 3. Histology and immunohistochemistry for GLT-1 in the mouse spinal cord at E13. $A-C$, Low-power micrographs of paraffin cross-sections stained with hematoxylin $(A)$ and by rabbit antiGLT-1 antibody ( $B$ and $C$ ). Immunostainings in the marginal zone/white matter (arrowheads) and in the dorsal horn $(D H)$ are seen in the wild-type mouse $(B)$, but not in the GLT-1(-/-) mouse $(C)$. $D$, High-power view of the ventral cord. GLT-1 is detected as tiny puncta in the marginal zone/white matter $(W M) . E, F$, Longitudinal cryostat sections stained by rabbit anti-GLT-1 antibody. GLT-1 is detected as longitudinal fibers running in the marginal zone/white matter (WM or arrowheads), from which transverse fibers with lower immunoreactivity enter the ventral horn $(V H)$. FP, Floor plate; $P$, pia mater; $R P$, roof plate. Asterisks indicate ventricular zone. Scale bars: $A-C, 100 \mu \mathrm{m} ; D, 10$ $\mu \mathrm{m} ; E, F, 100 \mu \mathrm{m}$.
$4 C$, red) and NSE (Fig. 4C, green) were both detected in the marginal zone and dorsal horn, yielding a fused yellow to orange color. At higher magnification, GLT-1 was overlapped with NSE but was more concentrated as tiny spots within larger NSEimmunoreactive structures (Fig. 4D). In the mantle zone, NSE was detected diffusely in neuronal perikarya as well, in which GLT-1 was sparsely distributed (Fig. 4C).

To clarify structural components in the marginal zone at E13, the lateral funiculus was examined by electron microscopy (Fig. $5 A$ ). In cross-sections, the marginal zone was occupied exclusively by round to irregular profiles variable in size, ranging from 0.04 to $2 \mu \mathrm{m}$. They contained various organelles, including smooth endoplasmic reticulum, clusters of vacuoles and vesicles, microtubules, intermediate filaments, and mitochondria. In general, larger profiles were irregular in shape and characterized by the cytoplasm with a fine filamentous meshwork and often had round structures limited by two concentric membranes (Fig. 5A, arrowheads), which have been referred to as "growth cone parcels" by Gorgels (1991a). On the other hand, smaller profiles were round to oval in shape and were relatively abundant in microtubules and intermediate filaments. In longitudinal sections, most, if not all, of the profiles in cross-sections turned out to be segments through axons forming frequent enlargements (data not shown). Axons were closely apposed to each other, having no junction-like specializations between them. Moreover, the cell membrane of axon profiles came in close contact with that of radial glial fibers (data not shown). By preembedding immunoelectron microscopy, GLT-1 was detected in some of the axon profiles (Fig. 5B). Within the labeled axons, immunoreaction products were not distributed evenly but were preferentially concentrated in only a small part of the axolemma. These immunoreactive patches were observed in axolemmal portions apposing adjacent axons and sometimes to shafts of radial glial fibers, but not to their end feet. No particular accumulations of organelles, such as vesicles, were observed beneath the labeled axolemma.
In cross-sections from E15 to P1, punctate labelings in the marginal zone/white matter were found in the ventral, lateral, and dorsal funiculi, particularly intensely in the lateral funiculus adjacent to the dorsal horn (Fig. 6A,B), but they disappeared from any regions of the white matter at $\mathrm{P} 7$ and thereafter (Fig. $6 C, E, G)$. Instead, weak immunoreactivity emerged in the mantle zone/gray matter at P1 (Fig. 6B), and the immunoreactivity showed a remarkable increase during the early postnatal period, peaking at P21 (Fig. 6C,G). In the spinal cord at P14, GLT-1 showed dense localization in neuropil regions, which surrounded immunonegative neuronal cell bodies and stem dendrites, and were also detected in fibrous structures traversing the white matter toward the pial surface (Fig. $6 C, E$ ). This pattern of immunostaining was very similar to that for GLAST (Fig. 6D). By double immunofluorescence, GLT-1 (Fig. 4E, green) was colocalized almost completely with GLAST (Fig. $4 E$, red) in reticular structures in the neuropil, yielding a fused yellow color. Furthermore, immunoelectron microscopy showed the presence of GLT-1 immunoreactivity in astrocytic processes, which entered elaborately between various neuronal elements to surround them (Fig. 6F).

\section{DISCUSSION}

In the present study, we have examined the expression of GLT-1 in the developing mouse spinal cord and disclosed distinct cellular and subcellular localizations in the period between fetal and postnatal stages.

During the fetal stages, we found contrasting distributions of GLT-1 mRNA and protein. The former was detected in the mantle zone/gray matter, whereas the latter was mainly in the marginal zone/white matter. From the unexpected distributions, we caref ully evaluated the authenticity of histochemical signals by confirming identical hybridization patterns with two antisense oligonucleotide probes and identical immunohistochemical patterns with four polyclonal antibodies. Recently, GLT-1 has also 
Figure 4. Images by confocal laser scanning microscopy. $A, B$, Double immunofluorescence for GLT-1 (green, rabbit antibody) and GLAST (red, guinea pig) in a paraffin cross-section at E13. GLT-1 immunoreactivity is seen in the marginal zone/white matter $(W M)$ of the ventral cord and in the dorsal horn $(D H)$, showing no overlaps with GLAST. A few transverse fibers (arrows) with GLT-1 immunoreactivity enter the ventral horn $(V H)$. $C, D$, Double immunofluorescence for GLT-1 (red, guinea pig) and NSE ( green, rabbit) in a cryostat cross-section at E13. Note their colocalization in the marginal zone/ white matter $(W M)$, but not in the ventral horn $(V H)$. E, Double immunofluorescence for GLT-1 ( green, rabbit) and GLAST (red, guinea pig) in a cross paraffin section at P14. $c$, Cell bodies; FP, floor plate; $P$, pia matter; $R P$, roof plate. Asterisks indicate the ventricular zone. Scale bars: $A, C, 50 \mu \mathrm{m} ; B, D, E, 10 \mu \mathrm{m}$.
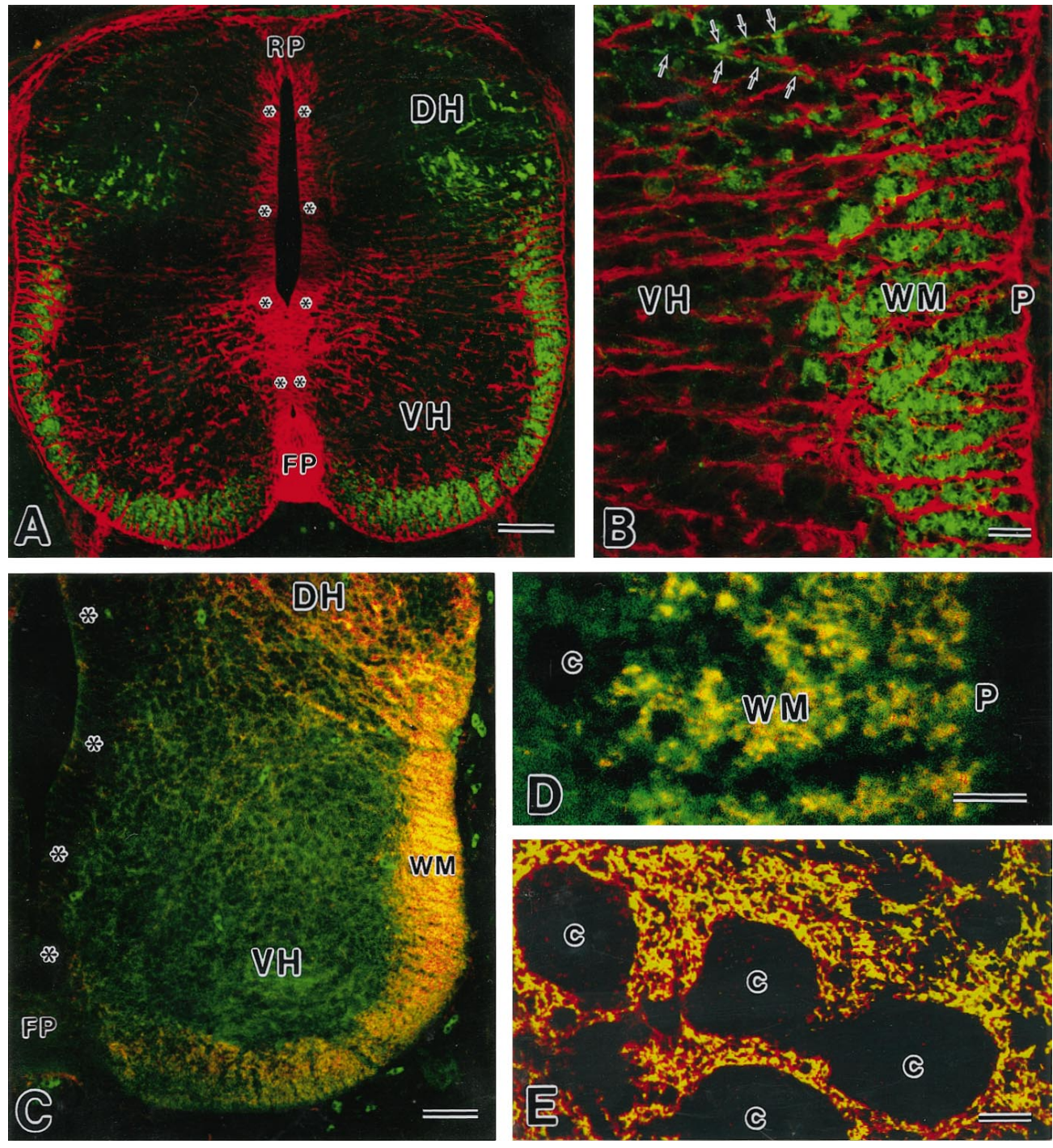

been reported to be present in white matter tracts of the developing rat CNS (Furuta et al., 1997).

\section{Transient neuronal expression of GLT-1}

In mice, motoneurons in the spinal ventral horn are generated at E9-E10.5, and dorsal horn neurons are generated at approximately E12 (Sims and Vaughn, 1979). Reflecting the active neurogenesis and successive migration, cell number in the mantle zone displays a remarkable increase between E11 and E13 (Shibata et al., 1997). At this period, GLT-1 and GLAST mRNAs show distinct distributions: the former in the mantle zone and the latter in the ventricular zone (compare Fig. $1 A, B$ in the present study with Fig. $2 A, B$ by Shibata et al., 1997). Using a specific antibody, we have shown previously that cells expressing GLAST at E11-E13 display morphological features characteristic of the radial glia, i.e., localization of cell bodies in the ventricular zone and radial fibers spanning the neural wall (Shibata et al., 1997). At E15, the cells begin to migrate to the mantle zone and to transform into astrocytes. Precursors of oligodendrocytes, which also exhibit the morphology of radial glia cells (Ono et al., 1997), are localized in the ventrobasal region of the rat spinal ventricular zone at E13 and E14 (Pringle and Richardson, 1993; Timsit et al., 1995). Therefore, the distribution of GLT-1 mRNA in the mantle zone at E11-E13 suggests its expression in a distinct cell popu- lation other than radial glial cells, most likely young neurons. Such a wide distribution of GLT-1 mRNA over the mantle zone has also been shown in the fetal mouse brain (Shibata et al., 1996; Sutherland et al., 1996).

\section{GLT-1 is localized at nonsynaptic sites of growing axons}

The marginal zone of the ventral cord at E13 was occupied exclusively by axons descending or ascending the cord. Compared with mature stages, these axons are characterized by extensive formation of enlargements, richness of organelles, and absence of glial ensheathment (except small contacts with radial glial fibers). These morphological features are common in many respects to axons in the rat corticospinal tract at approximately birth (De Kort et al., 1985; Gorgels, 1991a). GLT-1 was detected mainly in longitudinal fibers in the marginal zone at E13, when they were also immunopositive for NSE, a neuronal marker protein (Calker et al., 1978; Watanabe et al., 1993). In the mantle zone, on the other hand, GLT-1 was scarcely found except for a few transverse fibers running toward the marginal zone, whereas NSE was detected in neuronal cell bodies as well. These immunohistochemical results, together with the distribution of GLT-1 mRNA in the mantle zone, suggest that the GLT-1 protein synthesized in neuronal cell bodies is transported preferentially to growing 


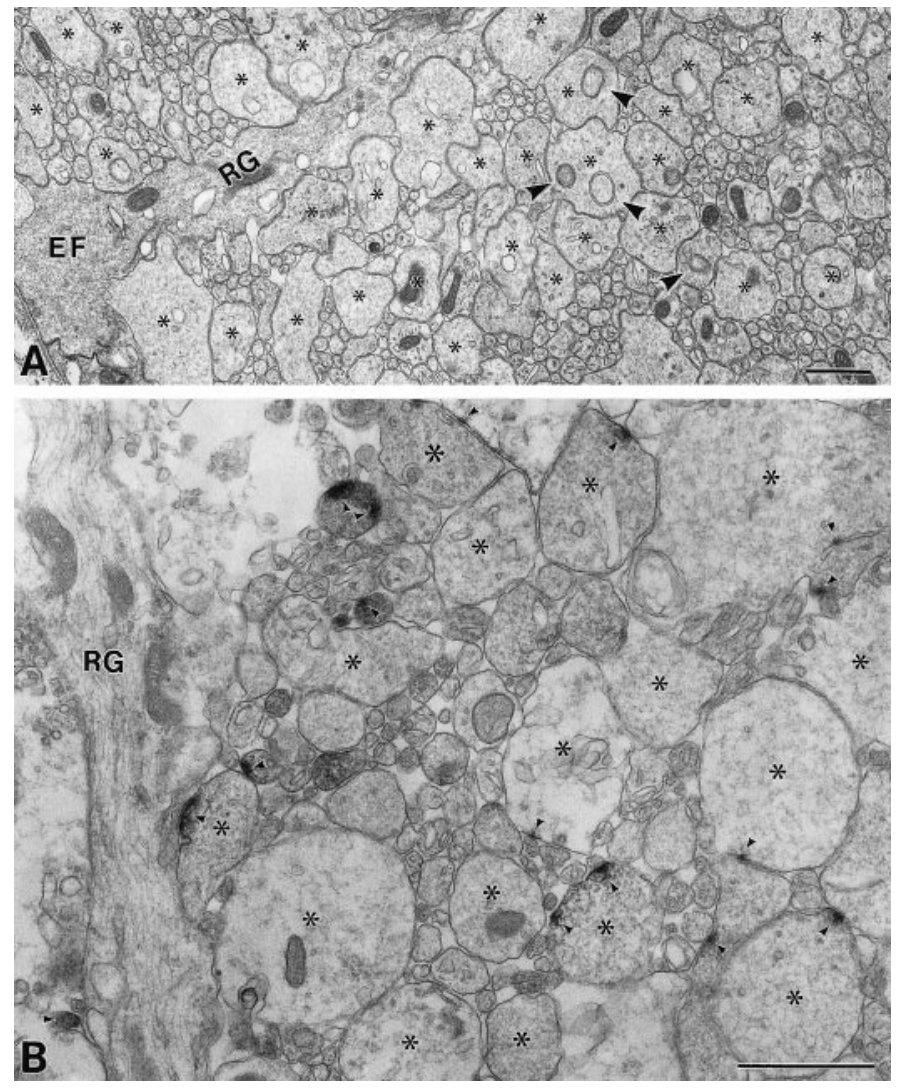

Figure 5. Electron micrographs of the spinal marginal zone at E13. $A$, Ultrastructure. Enlarged axonal portions are marked by asterisks. Arrowheads indicate growth cone parcels, which have been originally described for structures contained in growing pyramidal tract axons of neonatal rats (Gorgels, 1991a). B, Immunoelectron micrograph showing GLT-1. GLT-1 is detected in a small part of the axolemma apposing adjacent axons and sometimes to radial glial fibers (arrowheads). $E F$, End foot; $R G$, radial glial fiber. Scale bars, $1 \mu \mathrm{m}$.

axons. The axonal localization of GLT- 1 was confirmed by immunoelectron microscopy. In cross-sections through the white matter at P7, punctate labelings representing axonal localization disappeared. Therefore, GLT-1 is transiently expressed on immature axons growing out in white matter tracts.

GLT-1 exhibits a patch-like localization on the axolemma apposing other cellular elements. They are mostly neighboring axons and sometimes shafts of radial glial fibers. With such neuronal and glial elements, growing axons are known to form transient contacts having synapse-like junctional specializations (Henrikson and Vaughn, 1974; Vaughn et al., 1974, 1976; De Kort et al., 1985; Gorgels, 1991a,b). The specializations include a few clear vesicles in presynaptic axons, dense materials associated with presynaptic and postsynaptic membranes, and intercellular matrix in the synaptic cleft. In the spinal marginal zone of mice, the axoglial synapses are found at E13 and E14, but disappear thereafter (Henrikson and Vaughn, 1974). We also observed by electron microscopy some axoglial contacts with junction-like specialization at E13 (our unpublished observations), but they were much less frequent than GLT-1-immunopositive patches on the axolemma. Moreover, clear vesicles were not found in the vicinity of immunopositive patches. Therefore, it is safe to conclude that GLT-1 is localized primarily in nonspecialized axolemma apposing neighboring structures. However, the present immunoelectron microscopy with use of diffusible peroxidase substrate cannot exclude the possibility that some immunoreaction products, if not all, result from the diff usion from the original immunoreaction sites. In future studies, precise localization on growing axons needs to be determined by immunogold cytochemistry.

\section{Speculated role in glutamatergic signaling between growing axons}

Neuronal growth cones are highly motile enlargements and are thought to navigate a precise route through the developing nervous system and to recognize an appropriate synaptic partner. It is known that various neurotransmitters, including glutamate, have effects on growth cone movement (Mattson, 1988). Although the precise localization of glutamate receptors remains unknown at stages much earlier than synaptogenesis, transcripts of the receptor channel subunits are expressed (Monyer et al., 1991; Watanabe et al., 1992), and glutamate receptors are functionally active from early fetal stages (LoTurco et al., 1991). Recently, Owen and Bird (1997) have reported that the application of glutamate inhibits growth and motility of axons in cultured mouse spinal cord neurons, and the inhibitory actions are blocked by antagonist to the non-NMDA receptor, but not to the NMDA receptor. Zheng et al. (1996) have shown that growth cones of cultured Xenopus spinal cord neurons exhibit chemotropic turning responses for glutamate, and the response depends on the activation of the NMDA receptor. Glutamate transporters can either take in extracellular glutamate or release intracellular glutamate to the extracellular space, according to the electrochemical gradient across the cell membrane (Nicolls and Attwell, 1990). From the transient axonal localization at stages when growth cone-like axonal enlargements are actively formed, it is speculated that GLT-1 on growing axons subserves as a source of glutamate or its gradient to regulate the activation of glutamate receptors. Such a role of a transporter as the source of transmitters has been reported for GABA transporters on growth cones (Taylor and Gordon-Weeks, 1991) and has also been proposed for glutamate transporters on developing optic nerve axons (Chiu and Kriegler, 1994).

Homozygous mice defective in the GLT-1 gene are born at the frequency predicted by Mendelian ratios but suffer from spontaneous seizures leading to sudden death (Tanaka et al., 1997). In addition, these mice exhibit an abnormal posture, characterized by clasping the hindlimbs tightly, when the animals were lifted by the tail (our unpublished observations). Detailed neuroanatomical analyses should be challenged in future studies regarding development of white matter tracts, such as the corticospinal (pyramidal) tract.

\section{Switch to astrocytic expression at synaptogenic phase}

GLT-1 appeared in the gray matter at birth, and the intensity displayed a remarkable elevation during the early postnatal period. At P14, GLT-1 was densely distributed in the neuropil, observed as reticular stainings surrounding neuronal cell bodies and stem dendrites. Immunoelectron microscopy has demonstrated its localization in astrocytic processes surrounding synapses. Thus, it is evident that, although GLT-1 mRNA is continuously expressed in the mantle zone/gray matter, cells expressing the transcripts switch from neurons to astrocytes. As a result, GLT-1 becomes colocalized well with GLAST in astrocytic processes at P14. Compared with GLAST (Shibata et al., 1997), the appearance of reticular neuropilar staining is considerably late for GLT-1. Simultaneous with the onset of radial glial migration, the 

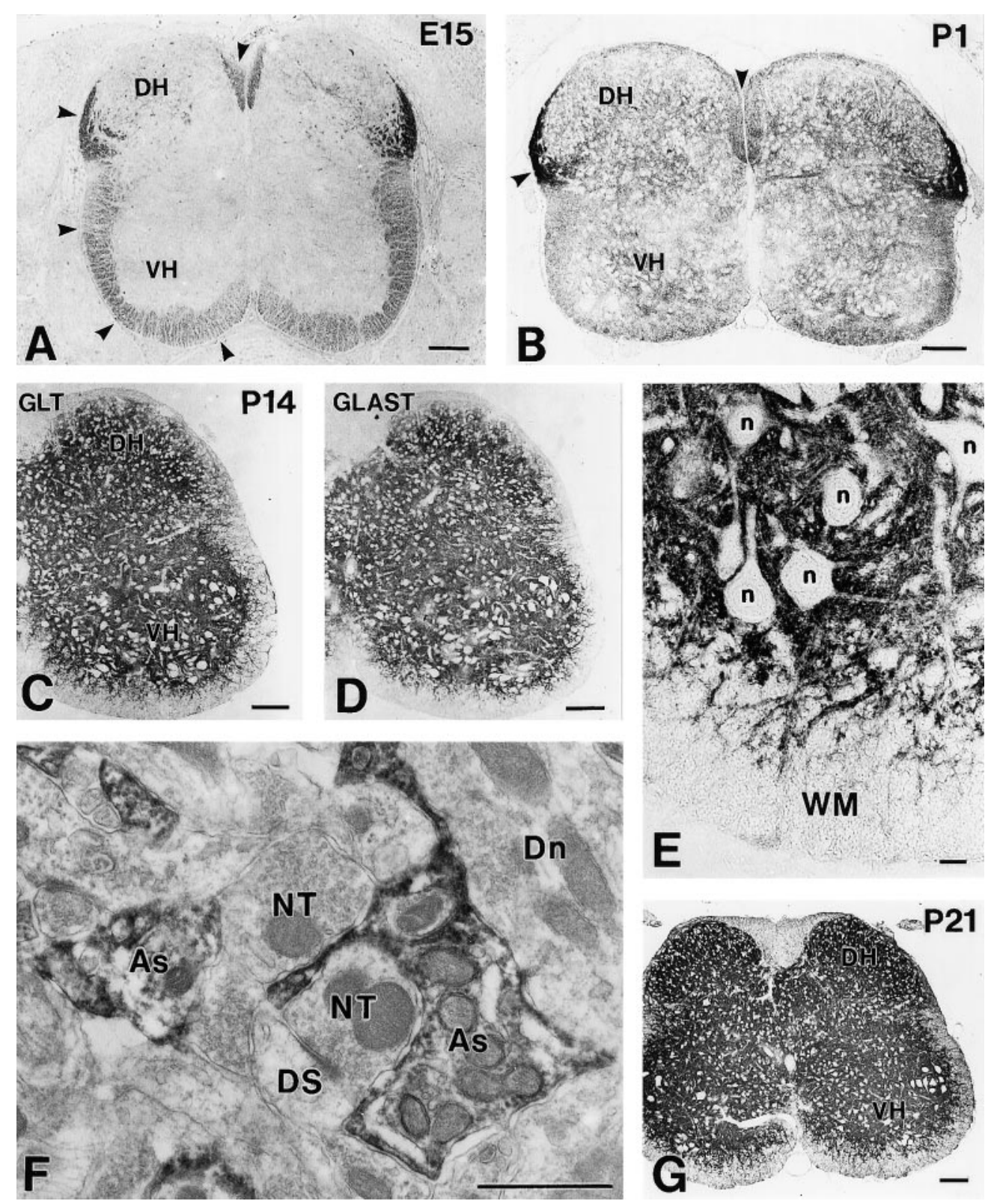

Figure 6. Spinal cords from E15 to P21. Cross paraffin sections were immunostained for GLT-1 (rabbit antibody) at E15 $(A), \mathrm{P} 1(B), \mathrm{P} 14(C)$, and $\mathrm{P} 21(G)$ and for GLAST at P14 (rabbit antibody) (D). Arrowheads indicate GLT-1 immunoreactivity in the marginal zone/white matter. $E$, High-power view of the ventral cord in $C$. F , Immunoelectron micrograph at P14 showing the localization of GLT-1 in astrocytic processes (As) surrounding immunonegative nerve terminals $(N T)$, dendritic spines $(D S)$, and dendrites $(D n) . D H$, Dorsal horn; $n$, neuronal cell bodies; $V H$, ventral horn; $W M$, white matter. Scale bars: $A-D, G$, $100 \mu \mathrm{m} ; E, 10 \mu \mathrm{m} ; F, 1 \mu \mathrm{m}$.

transformation into astrocytes begins at E15, when tiny membranous protrusions having GLAST immunoreactivity appear around radial fibers (Shibata et al., 1997). During the late fetal and early postnatal periods, the tiny protrusions progressively increase with concomitant reduction and disappearance of radial fiber staining. Therefore, GLT-1 becomes recruited on preexisting astrocytic processes during the early postnatal period. The relatively late appearance of GLT-1 in astrocytes has also been reported in the rat brain (Ullensvang et al., 1997). In the adult, levels of GLT-1 and GLAST are differentially downregulated in astrocytes of different neural regions, thus establishing their distinct spatial distributions in the mature CNS (Rothstein et al., 1994; Torp et al., 1994; Lehre et al., 1995; Shibata et al., 1996). From these results, dense colocalization of GLT-1 and GLAST at early postnatal stages may suggest increasing demands for astrocytic glutamate transporters at synaptogenic stages.

In conclusion, it is now clear that GLT-1 and GLAST are localized first on growing axons and radial glial fibers, respectively, and later on astrocytic processes. Considering that the activation of glutamate receptors affects radial glia-guided neuronal migration (Rakic, 1971; Komuro and Rakic, 1993) and growth cone movement, it is interesting to think that the two glutamate transporters might play cooperative and complementary roles in neural development, as they do in rapid clearance of synaptically released glutamate to protect mature neurons from excitotoxicity.

\section{REFERENCES}

Calker DV, Müller M, Hamprecht B (1978) Neuron-specific enolase is a molecular marker for peripheral and central neuroendocrine cells. Nature 276:834-841.

Chaudhry FA, Lehre KP, van Lookeren Campagne M, Ottersen OP, Danbolt NC, Storm-Mathisen J (1995) Glutamate transporters in glial plasma membranes: highly differentiated localizations revealed by quantitative ultrastructural immunocytochemistry. Neuron 15:711-720.

Chiu SY, Kriegler S (1994) Neurotransmitter-mediated signaling between axons and glial cells. Glia 11:191-200.

Choi BH (1981) Radial glia of developing human fetal spinal cord: golgi, immunohistochemical and electron microscopic study. Dev Brain Res $1: 249-267$. 
Choi DW (1992) Excitotoxic cell death. J Neurobiol 23:1261-1276.

De Kort EJM, Gribnau AAM, van Aanholt HTH, Nieuwenhuys R (1985) On the development of the pyramidal tract in the rat. I. The morphology of the growth zone. Anat Embryol 172:195-204.

Furuta A, Rothstein JD, Martin LJ (1997) Glutamate transporter protein subtypes are expressed differentially during rat CNS development. J Neurosci 17:8363-8375.

Gorgels TGMF (1991a) Outgrowth of the pyramidal tract in the rat cervical spinal cord: growth cone ultrastructure and guidance. J Comp Neurol 306:95-116.

Gorgels TGMF (1991b) Junctional specializations between growth cones and glia in the developing rat pyramidal tract: synapse-like contacts and invaginations. J Comp Neurol 306:117-128.

Henrikson CK, Vaughn JE (1974) Fine structural relationships between neurites and radial glial processes in developing mouse spinal cord. J Neurocytol 3:659-675.

Hertz L (1979) Functional interactions between neurons and astrocytes. I. Turnover and metabolism of putative amino acid transmitters. Prog Neurobiol 13:277-323.

Kanai Y, Hediger MA (1992) Primary structure and functional characterization of a high-affinity glutamate transporter. Nature 360:467-471.

Kanai Y, Trotti D, Nussberger S, Hediger MA (1997) The high affinity glutamate transporter family: structure, function, and physiological relevance. In: Neurotransmitter transporters: structure, function, and regulation (Reith MEA, ed), pp 171-213. Totowa, NJ: Humana.

Komuro H, Rakic P (1993) Modulation of neuronal migration by NMDA receptors. Science 260:95-98.

Lehre KP, Levy LM, Ottersen OP, Storm-Mathisen J, Danbolt NC (1995) Differential expression of two glial glutamate transporters in the rat brain: quantitative and immunocytochemical observations. J Neurosci 15:1835-1853.

LoTurco JL, Blanton MG, Kriegstein AR (1991) Initial expression and endogenous activation of NMDA channels in early neocortical development. J Neurosci 11:792-799.

Mattson MP (1988) Neurotransmitters in the regulation of neuronal cytoarchitecture. Brain Res Rev 13:179-212.

Mayer BL, Westbrook GL (1987) The physiology of excitatory amino acids in the vertebrate central nervous system. Prog Neurobiol 28:197-276.

Monaghan DT, Bridges RJ, Cotman CW (1989) The excitatory amino acid receptors: their classes, pharmacology and distinct properties in the central nervous system. Annu Rev Pharmacol Toxicol 29:365-402.

Monyer H, Seeburg PH, Wisden W (1991) Glutamate-operated channels: developmentally early and mature forms arise by alternative splicing. Neuron 6:799-810.

Mukainaka Y, Tanaka K, Hagiwara T, Wada K (1995) Molecular cloning of two glutamate transporter subtypes from mouse brain. Biochim Biophys Acta 1244:233-237.

Nicholls D, Attwell D (1990) The release and uptake of excitatory amino acids. Trends Pharmacol Sci 11:462-468.

Ono K, Fujisawa H, Hirano S, Norita M, Tsumori T, Yasui Y (1997) Early development of the oligodendrocyte in the embryonic chick metencephalon. J Neurosci Res 48:212-225.

Owen AD, Bird MM (1997) Role of glutamate in the regulation of the outgrowth and motility of neurites from mouse spinal cord neurons in culture. J Anat 191:301-307.

Pringle NP, Richardson WD (1993) A singularity of PDGF alphareceptor expression in the dorsoventral axis of the neural tube may define the origin of the oligodendrocyte lineage. Development 117:525-533.

Raff MC, Millar RH, Noble M (1983) A glial progenitor cell that develops in vitro into an astrocyte or an oligodendrocyte depending on culture medium. Nature 303:390-396.

Rakic P (1971) Guidance of neurons migrating to the fetal monkey neocortex. Brain Res 33:471-476.

Rothstein JD, Martin L, Levey AI, Dykes-Hoberg M, Jin L, Wu D, Nash N, Kuncl RW (1994) Localization of neuronal and glial glutamate transporters. Neuron 13:713-725.
Shibata T, Watanabe M, Tanaka K, Wada K, Inoue Y (1996) Dynamic changes in expression of glutamate transporter mRNAs in developing brain. NeuroReport 7:705-709.

Shibata T, Yamada K, Watanabe M, Ikenaka K, Wada K, Tanaka K, Inoue Y (1997) Glutamate transporter GLAST is expressed in the radial glia-astrocyte lineage of developing mouse spinal cord. J Neurosci 17:9212-9219.

Sims TJ, Vaughn JE (1979) The generation of neurons involved in an early reflex pathway of embryonic mouse spinal cord. J Comp Neurol 183:707-720.

Storck T, Schulte S, Hofmann K, Stoffel W (1992) Structure, expression, and functional analysis of a $\mathrm{Na}^{+}$-dependent glutamate/aspartate transporter from rat brain. Proc Natl Acad Sci USA 89:10955-10959.

Sutherland ML, Delaney TA, Noebels JL (1996) Glutamate transporter mRNA expression in proliferative zones of the developing and adult murine CNS. J Neurosci 16:2191-2207.

Tanaka K (1993) Cloning and expression of a glutamate transporter from mouse brain. Neurosci Lett 159:183-186.

Tanaka K, Watase K, Manabe T, Yamada K, Watanabe M, Takahashi K, Iwama H, Nishikawa T, Ichihara N, Kikuchi T, Okuyama S, Kawashima N, Hori S, Takimoto M, Wada K (1997) Epilepsy and exacerbation of brain injury in mice lacking the glutamate transporter GLT-1. Science 276:1699-1702.

Taylor J, Gordon-Weeks P (1991) Calcium-independent gammaaminobutyric acid release from growth cones: role of gammaaminobutyric acid transporter. J Neurochem 56:273-280.

Timsit S, Martinez S, Allinquant B, Peyron F, Puelles L, Zalc B (1995) Oligodendrocytes originate in a restricted zone of the embryonic ventral neural tube defined by DM-20 mRNA expression. J Neurosci 15:1012-1024.

Torp R, Danbolt NC, Babaie E, Bjørås M, Seeberg E, Storm-Mathisen J, Ottersen OP (1994) Differential expression of two glial glutamate transporters in the rat brain: an in situ hybridization study. Eur J Neurosci 6:936-942.

Ullensvang K, Kehre KP, Storm-Mathisen J, Danbolt NC (1997) Differential developmental expression of the two rat brain glutamate transporter protein GLAST and GLT. Eur J Neurosci 9:1646-1655.

Vaughn JE, Henrikson CK, Grieshaber JA (1974) A quantitative study of synapses on motor neuron dendritic growth cones in developing mouse spinal cord. J Cell Biol 60:664-672.

Vaughn JE, Henrikson CK, Wood JG (1976) Surface specializations of neurites in embryonic mouse spinal cord. Brain Res 110:431-445.

Watanabe M, Sakimura K, Takahashi Y, Kondo H (1990) Ontogenic changes in expression of neuron-specific enolase (NSE) and its mRNA in the Purkinje cells of the rat cerebellum: immunohistochemical and in situ hybridization study. Dev Brain Res 53:89-96.

Watanabe M, Inoue Y, Sakimura K, Mishina M (1992) Developmental changes in distribution of NMDA receptor channel subunit mRNAs. NeuroReport 3:1138-1140.

Watanabe M, Nagamine T, Sakimura K, Takahashi Y, Kondo H (1993) Developmental study of the gene expression for $\alpha$ and $\gamma$ subunits of enolase in the rat brain by in situ hybridization histochemistry. J Comp Neurol 327:350-358.

Watanabe M, Fukaya M, Sakimura K, Manabe T, Mishina M, Inoue Y (1998) Selective scarcity of NMDA receptor channel subunits in the stratum lucidum (mossy fiber-recipient layer) of the mouse hippocampal CA3 subfield. Eur J Neurosci 10:478-487.

Watase K, Hashimoto K, Kano M, Yamada K, Watanabe M, Inoue Y, Okuyama S, Sakagawa T, Ogawa S, Kawashima N, Hori S, Takimoto M, Wada K, Tanaka K (1998) Motor discoordination and increased susceptibility to cerebellar injury in GLAST mutant mice. Eur J Neurosci 10:976-988.

Yamada K, Watanabe M, Shibata T, Tanaka K, Wada K, Inoue Y (1996) EAAT4 is a post-synaptic glutamate transporter at Purkinje cell synapses. NeuroReport 7:2013-2017.

Zheng JQ, Wan J-j, Poo M-m (1996) Essential role of filopodia in chemotropic turning of nerve growth cone induced by a glutamate gradient. J Neurosci 16:1140-1149. 